November 11, 2000

\title{
Bound States from Regge Trajectories in a Scalar Model
}

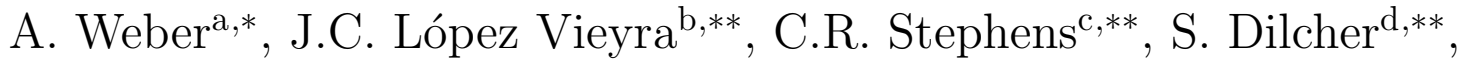 \\ P.O. Hess'印** \\ *Instituto de Física y Matemáticas, UMSNH, 58040 Morelia, Michoacán, Mexico, \\ ${ }^{* *}$ Instituto de Ciencias Nucleares, UNAM, 04510 México D.F., Mexico
}

\begin{abstract}
The calculation of bound state properties using renormalization group techniques to compute the corresponding Regge trajectories is presented. In particular, we investigate the bound states in different charge sectors of a scalar theory with interaction $\phi^{\dagger} \phi \chi$. The resulting bound state spectrum is surprisingly rich. Where possible we compare and contrast with known results of the Bethe-Salpeter equation in the ladder approximation and, in the non-relativistic limit, with the corresponding Schrödinger equation.
\end{abstract}

\footnotetext{
${ }^{\dagger}$ This work was supported by Conacyt grants 3298P-E9608 and 3298P-32729-E and also CIC-UMSNH.

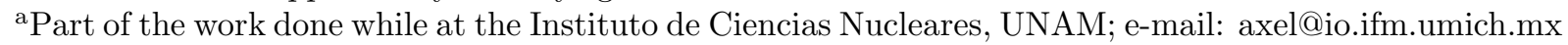

be-mail: vieyra@pythia.nuclecu.unam.mx

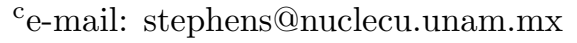

${ }^{\mathrm{d}}$ Supported during part of the work by fellowships of the DAAD and the Mexican Government; present address: Fakultät für Physik, A. Ludwig Universität, 79104 Freiburg, Germany; e-mail: dilcher@physik.unifreiburg.de

ee-mail: hess@nuclecu.unam.mx
} 


\section{Introduction}

The formation of bound states from more fundamental constituents is one of the most important phenomena in physics spanning a vast spectrum of energy scales, ranging from high energies, associated with QCD bound states and resonances, to low energies in, for example, superconductivity. In describing completely the crossover between bound states and their constituents one has to address two fundamental problems: i) the formation of a single bound state in terms of its more fundamental constituents, and ii) the many-body collective effects associated with many bound states. The former has traditionally been attacked by way of the Bethe-Salpeter equation [1] (for an early review see ref. [2]) and the latter by using effective field theory, as in the Ginzburg-Landau formulation of superconductivity for instance.

The problems with the Bethe-Salpeter equation are well known and various, especially when it comes to having controlled, systematic approximation schemes that preserve all relevant symmetries. On the other hand, with effective field theory one loses contact with the original fundamental degrees of freedom. Naturally, any technique which could give fresh insight into either of these two aspects of the bound state problem would be more than welcome.

Given the non-perturbative nature of the underlying problem one might expect a nonperturbative tool such as the renormalization group $(\mathrm{RG})$ to be useful. The fundamental problem here, in terms of a Wilsonian coarse graining picture, is to formulate an RG that coarse grains bound states at low energies and their fundamental constituents at high energies. Some progress has been made in this direction [3]. The fundamental problem however remains. In the more restricted case of a non-relativistic system a non-relativistic effective field theory description allied to an RG strategy that isolates contributions from relativistic and non-relativistic momentum scales has been used to calculate quantities such as the hyperfine structure of atoms such as muonium or positronium [4].

In this paper we present a new and different approach to the description of bound states based on RG techniques. Specifically, we exploit the indirect access that Regge theory gives to the calculation of bound state properties. Regge theory is unique in bringing together two extremely different kinematical regions. Given the asymmetric behaviour of the two-particle scattering amplitude in the "Regge limit", $t \rightarrow \infty, s$ fixed (where $s$ and $t$ are the Mandelstam variables), it predicts the energy spectrum of the bound states in the theory. .

Given the difficulty in predicting the existence and properties of bound states, if the theoretical determination of Regge trajectories from the asymptotic behaviour of the scattering amplitudes should turn out to be more accessible, we may be able to devise a more convenient approach to their exploration. Indeed, in spite of the well known relation between bound states and asymmetric scattering there has been little done in terms of computing bound state properties in relativistic systems using calculated Regge trajectories. In this sense the present paper serves as a feasibility proof that it is in fact possible to study bound state properties, both qualitatively and quantitatively, via Regge trajectories. Of course, it should be mentioned that Regge trajectories also play a prominent rôle in the description of

\footnotetext{
${ }^{1} \mathrm{~A}$ brief review of Regge theory, tailored to our present purposes, has been given in ref. [5]. A more complete presentation may be found in ref. [6].
} 
high energy processes in QCD in the experimentally important kinematical region $x \ll 1$ (for recent data see ref. [7]). In the present contribution, however, we will concentrate exclusively on the bound state aspects of the theory.

Regge trajectories were originally calculated in quantum field theory by a direct summation of leading logs (in $t$ ) [8] and, again, using the Bethe-Salpeter equation [9]. There was a renewal of interest in Regge trajectories associated with the BKFL equation [10], which recently has been solved to next to leading order [11]. In ref. [5] we presented a new methodology based on RG methods for doing field theory calculations in the Regge limit. It has been sometimes thought that the RG was incapable of accessing the Regge limit. As we have explained in some detail in ref. [5], this is partially due to a somewhat restrictive view of what the $\mathrm{RG}$ is and does. Indeed, a generalization in the spirit of "environmentally friendly" renormalization [12] turned out to be necessary for that purpose.

One of the major disadvantages associated with the summation of leading logs and the Bethe-Salpeter equation in comparison with RG methods lies in the difficulty of incorporating higher-order corrections in a systematic and consistent way. In very simple cases, such as $\phi^{3}$, an analysis of the combinatorics of the $n$-loop diagrams is not so difficult. However, as soon as one treats more complicated, i.e. realistic, theories, the $\mathrm{RG}$ may be expected to be superior to other methods. Even in the case of the simple looking $\phi^{\dagger} \phi \chi$ scalar theory considered in ref. [5], a summation of the leading logs by hand looks forbidding. Only $a$ posteriori have we been able to verify by a recursive argument that the direct summation leads to the same result as the RG, and also the Bethe-Salpeter equation.

In this paper we will use our methods to calculate the bound state spectrum in a scalar theory. We will compare our results to those of the Bethe-Salpeter equation where possible and discuss the relative merits of the different techniques.

\section{Wick-Cutkosky Model}

To illustrate the methodology we consider a theory consisting of a complex scalar field $\phi$ of mass $m$, to which we assign charge one, and a real scalar field $\chi$ of mass $\mu$, with interaction $g \phi^{\dagger} \phi \chi$. The reason we choose this theory is that it is devoid of complications due to gauge fields and spin yet exhibits a highly non-trivial and rich bound state spectrum. In [5] we investigated the asymptotic behaviour of the two-particle scattering amplitude $A(s, t)$ in this theory, where $s$ and $t$ are the standard Mandelstam variables. In the limit of large $t$ we found different types of asymptotic behaviour, corresponding to a double charged, a single charged and a neutral state in the $s$-channel. Other processes are related by charge conjugation symmetry.

In this section, we consider the simplest of these cases, namely the charge two $(Q=$ 2) sector corresponding to a double charged particle exchanged in the s-channel. In ref. [5] we found for the leading even-signature Regge trajectory to one-loop order (after RG improvement)

$$
\alpha_{2}^{+}(s)=g^{2} K_{m m}(s)-1,
$$

\footnotetext{
${ }^{2}$ Strictly speaking, the exchanged object is a Reggeon. Only in the case where its angular momentum takes on a physical value can the object be identified with a physical particle, be it a bound state or a resonance.
} 
where the function $K$ is

$$
K_{m_{1} m_{2}}(s)=\frac{1}{4 \pi^{2}} \frac{\arctan \sqrt{\frac{s-\left(m_{1}-m_{2}\right)^{2}}{\left(m_{1}+m_{2}\right)^{2}-s}}}{\sqrt{\left(s-\left(m_{1}-m_{2}\right)^{2}\right)\left(\left(m_{1}+m_{2}\right)^{2}-s\right)}} .
$$

The leading log contributions resummed by the trajectory (1) come from ladder diagrams with $\phi$-particles on the sides and $\chi$-particles on the rungs. The function $K$ corresponds to a two-dimensional bubble diagram resulting from the contraction of the "d-lines" (rungs of the ladder) and the corresponding dimensional reduction in the Regge limit.

Due to the Bose statistics of the $\phi$ field there is no odd-signature trajectory. However, we can easily generalize this result to the case of two different charged particles with masses $m_{1}$ and $m_{2}$. The leading trajectories of even and odd signature are then given (to one loop) by

$$
\alpha_{W}(s)=g^{2} K_{m_{1} m_{2}}(s)-1
$$

This result for the Regge trajectory has been known (in the case of equal masses) for a long time [8, 9]. Physically, the (exact) trajectory is expected to describe the bound state spectrum of the Wick-Cutkosky model [13] via

$$
\alpha_{W}\left(M^{2}\right)=l
$$

according to Regge's theory. Here $M$ is the mass of the bound state with spin $l, l=0,1,2, \ldots$. Surprisingly enough, we were not able to find a discussion of the bound state spectrum following from (四) anywhere in the literature.

Before discussing eq. (4) several remarks are in order. First of all, we use the term "Wick-Cutkosky model" somewhat indiscriminately, regardless of the mass of the exchanged $\chi$-particle, and refering in principle to the solution of the full Bethe-Salpeter equation for the bound state problem rather than some approximation to it. However, it is only in the massless case and in the ladder approximation that the Bethe-Salpeter equation has been analytically solved [13], and this special case constitutes the proper Wick-Cutkosky model. Both the ladder approximation to the Bethe-Salpeter equation and the one-loop approximation to the Regge trajectory are supposed to reproduce the spectrum properly only if the coupling $g$ is not too large. Furthermore, it should be noted that eq. (4) is not expected to give the complete spectrum of the theory, but rather the lowest mass eigenvalue to every spin $l$. Finally, there is no dependence on the mass of the exchanged $\chi$-particle in the one-loop approximation to the Regge trajectory as a result of taking the limit $t \rightarrow \infty$. On the other hand, the ladder approximation does display such a dependence. In other words, the one-loop approximation to the Regge trajectory does not distinguish between a Coulomb-like and a Yukawa-like potential. This appears to be a serious restriction for the phenomenological application of the Regge formalism and will be discussed in more detail in the following. One should, however, bear in mind that for the most interesting theories (namely gauge theories) most of the exchanged particles are massless. 
Let us now solve eq. (田) for the bound state mass $M$. To this end, it is convenient to introduce the dimensionless variables

$$
e^{2}=\frac{g^{2}}{16 \pi m_{1} m_{2}}
$$

a combination that is commonly used as the effective coupling in the Wick-Cutkosky model, and

$$
y=\frac{\left(m_{1}+m_{2}\right)^{2}-\left(m_{1}-m_{2}\right)^{2}}{\left(m_{1}+m_{2}\right)^{2}-M^{2}} .
$$

The physical meaning of the latter quantity will become apparent below. Note that we here explicitly exclude the cases $m_{1}=0$ and $m_{2}=0$. In the limit where $m_{1} \rightarrow 0$ or $m_{2} \rightarrow 0$ a perturbative expansion of the Regge trajectories will break down due to the presence of infrared divergences. In this limit a further renormalization of the Regge trajectory itself will be necessary. We will not discuss this issue further here.

Eq. (4) now takes the form

$$
\frac{e^{2}}{\pi} y \frac{\arctan \sqrt{y-1}}{\sqrt{y-1}}=l+1 .
$$

Solving eq. (7) we compare, over the entire range $M=0$ to $M=m_{1}+m_{2}$, with known results from the ladder approximation to the Bethe-Salpeter equation for the case $\mu=0$, $m_{1}=m_{2}=m$, and $l=0$ (the ground state) [14. The comparison can be seen in fig. 1 where we also plot the Bethe-Salpeter results for $\mu=m / 10$. There are two noteworthy features: first of all the difference between our result and that of ref. [14, although relatively large, cannot be used to favour one methodology versus the other as the difference appears in the region of rather strong coupling $\left(e^{2} \gtrsim 0.5\right.$, see also fig. 2 below). In this strong-coupling region higher loop corrections will be important. We believe that such corrections are easier to compute in the present RG approach than in the Bethe-Salpeter equation. The second feature is that, as one can see from comparing the two Bethe-Salpeter curves, the effect of $\mu \neq 0$ is relatively weak, which leads us to believe that our methodology, based on the Regge limit, should not suffer too much from the fact that, at least at lowest order, our results are $\mu$-independent. The $\mu$-dependence is, however, significant near the threshold $M=2 m$.

We can gain a qualitative understanding of the situation from a simple phenomenological consideration: a rough estimate for the extension of the bound state is given by the Bohr radius

$$
a_{l}=\frac{l+1}{m_{r d} e^{2}}
$$

for states with principal quantum number $n=l+1$. Here $m_{r d}$ is the usual reduced mass

$$
m_{r d}=\frac{m_{1} m_{2}}{m_{1}+m_{2}}
$$

Intuitively, for a state to remain bound, its radius cannot be larger than the range of the potential, hence $a_{l} \lesssim R=1 / \mu$, or

$$
e^{2} \gtrsim \frac{\mu}{m_{r d}}(l+1)
$$




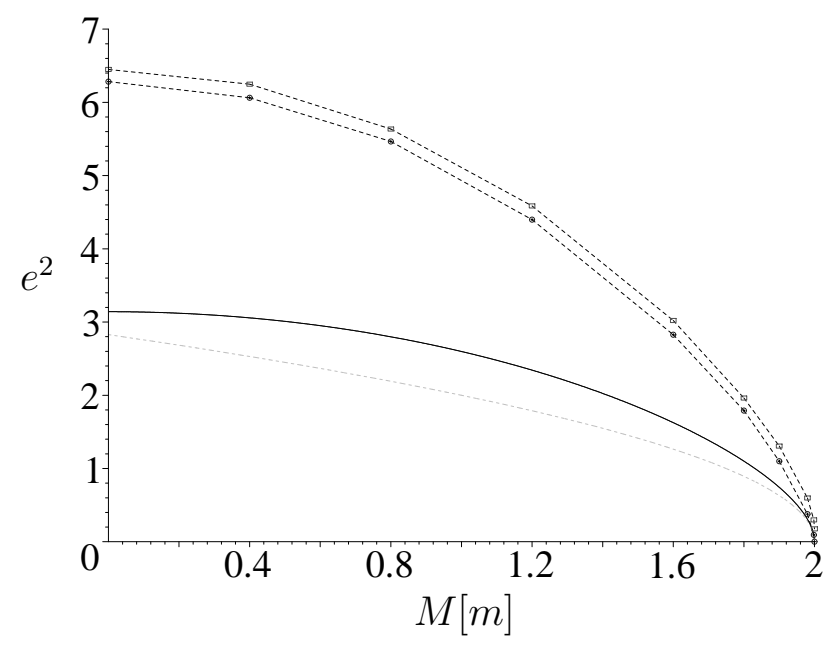

Figure 1: Graph of coupling versus mass of the ground state for $m_{1}=m_{2}=m$. The solid line is from equation (7) and the dotted line from the non-relativistic Coulomb spectrum (with $M=m_{1}+m_{2}+E$ ). The dashed curves represent the (numerical) solutions [14] of the Bethe-Salpeter equation, with $\mu=0$ for the lower dashed curve and $\mu=m / 10$ for the upper one.

In the case of a Yukawa-like potential $(\mu \neq 0)$ this yields an estimate of the number of existing bound states (with $n=l+1$ ), while for $\mu=0$ there is obviously no restriction.

Similarly, the finiteness of $\mu$ or the range of the potential is not expected to be significant for the spectrum as long as $a_{l} \ll R$, or equivalently

$$
e^{2} \gg \frac{\mu}{m_{r d}}(l+1) \text {. }
$$

We should expect our $\mu$-independent results to be quantitatively correct in this region (except for corrections from higher loop orders). In fact, this expectation is borne out by fig. 1, where (11) becomes $e^{2} \gg 0.2$ for $\mu=m / 10$, and in this region the deviation from the $\mu=0$ curve is indeed small.

In the following, we will often consider the non-relativistic limit, simply because it is relatively easy to obtain exact solutions in this limit. A system will be considered nonrelativistic whenever $p \ll m_{r d}$, where we can obtain an estimate for the momentum from Heisenberg's uncertainty relation, $p \approx 1 / a_{l}$. With $(8)$, the condition for a system to be non-relativistic becomes

$$
e^{2} \ll l+1
$$

Thus from (10) there can be non-relativistic bound states only if

$$
\mu \ll m_{r d} \text {. }
$$

Let us now consider the solutions of (4) in the weak-coupling or non-relativistic (for all l) limit $e^{2} \ll 1$. Eq. (7) implies that in this case the solutions satisfy $y \gg 1$. Consequently,

$$
\frac{\left(m_{1}+m_{2}\right)^{2}-M^{2}}{2\left(m_{1}+m_{2}\right)} \ll 2 m_{r d}<m_{1}+m_{2},
$$



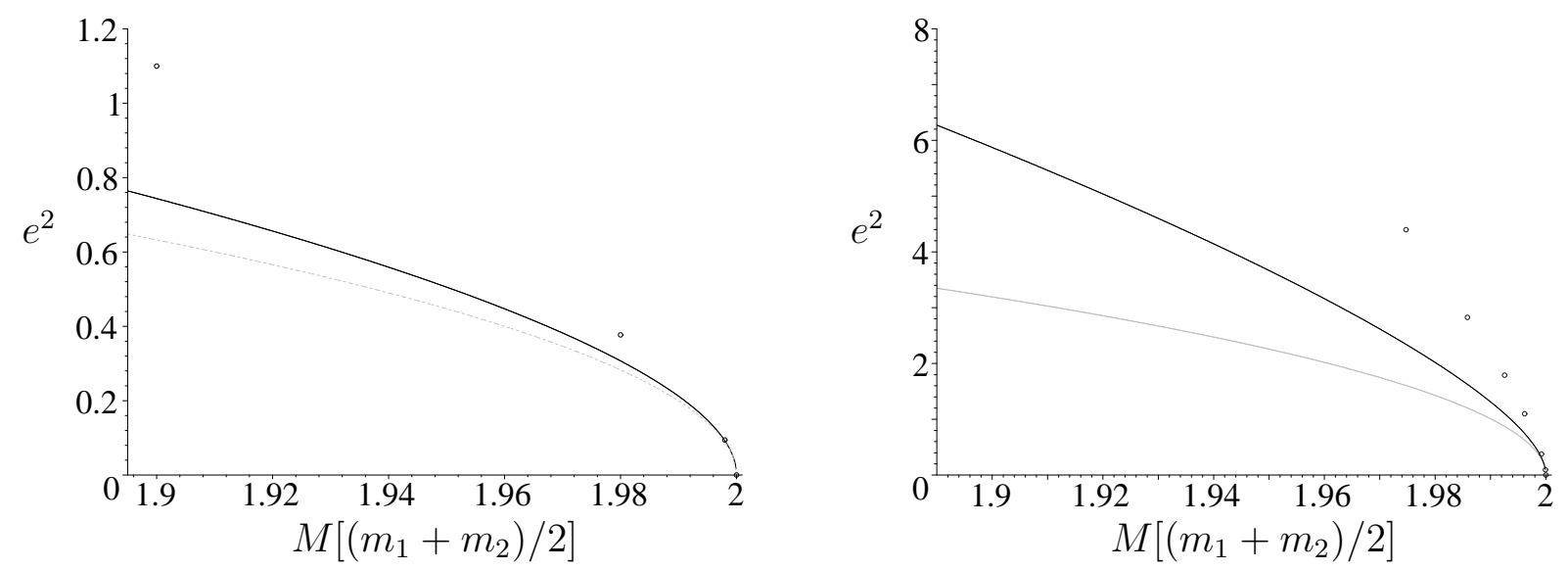

Figure 2: Graph of coupling versus mass of the ground state for $m_{1}=m_{2}, \mu=0$ (left) and $m_{1}=100 m_{2}, \mu=0$ (right), respectively. The solid lines are from equation (7); the dashed curves are from the non-relativistic Coulomb spectrum and the points from (numerical) solutions [14] of the Bethe-Salpeter equation.

where $m_{r d}$ is the reduced mass defined in (9). We can then identify the left-hand side of (14) with the absolute value of the (negative) binding energy $E$, considering that this definition is equivalent to

$$
M=m_{1}+m_{2}+E
$$

in the limit $|E| \ll m_{1}+m_{2}$.

Replacing $y$ by $2 m_{r d} /|E|$ and considering the limit $y \gg 1$, the solution to (17) is easily found to be

$$
E=-\frac{m_{r d} e^{4}}{2(l+1)^{2}},
$$

the well-known non-relativistic Coulomb spectrum (with principal quantum number $n=$ $l+1)$.

The Bethe-Salpeter equation for a massless $\chi$ particle in the ladder approximation leads, of course, to the same result in the limit $e^{2} \rightarrow 0$ [13]. For massive $\chi$, however, the threshold behavior is qualitatively different: there exists a critical coupling $e_{c}^{2}$ below which there are no bound states. In the limit $\mu \rightarrow 0$, one has $e_{c}^{2} \cong 0.84\left(\mu / m_{r d}\right)$ [15 which is in excellent agreement with the estimate (10). The existence of a critical coupling is not accounted for by the one-loop formula for the Regge trajectory. However, as soon as $e^{2} \gg\left(\mu / m_{r d}\right)$, the variation of the ground state energy with $\mu$ will be quantitatively small.

In fig. 2 we compare the solution of eq. (7) in the non-relativistic region with eq. (16) and once again the results of [14] using the ladder approximation for $m_{1}=m_{2}$ and $m_{1}=100 m_{2}$, and $\mu=0$. Since we have not found any remark in the literature, let us note that the numerical values for the coupling constants near threshold given in ref. [14 for the unequal mass case are in error (or the error in the numerical computations is much larger than 
stated). 5 We have produced the data presented in fig. 2 by applying the Wick-Cutkosky transformation [2, 13] to the equal mass results given in ref. [14]. Obviously, the Regge data lie in between the Bethe-Salpeter and the non-relativistic curves. This is a welcome result in so far as virtually all alternative approaches to relativistic bound state calculations yield values in between these two curves.

\section{Charge One Bound States}

In the case of the exchange of a Reggeon with charge one in the s-channel, we found in ref. [5] the following trajectories

$$
\alpha_{1}^{ \pm}(s)= \pm g^{2} K_{m \mu}(s)-1
$$

The positive-signature trajectory $\alpha_{1}^{+}$corresponds to an attractive potential, while the negative-signature trajectory $\alpha_{1}^{-}$indicates a repulsive interaction. Consequently, $\alpha_{1}^{+}$leads to a series of bound states, while $\alpha_{1}^{-}$always has a negative real part (for real values of $s$ ).

The appearance of a repulsive interaction (through exchange of spinless bosons) is a known feature of exchange interactions. The physics of an exchange interaction is clearly illustrated by the diagrams that contribute to leading order to the two trajectories and which have the following typical appearance

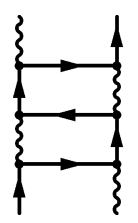

The bound state spectrum generated by the trajectory $\alpha_{1}^{+}$is identical to the one obtained from $\alpha_{W}$ in the previous section, with $m_{1}$ and $m_{2}$ replaced by $m$ and $\mu$, respectively, in the definition of the effective coupling and the reduced mass, except for the fact that $l$ can take only even values. This is the spectrum one would expect for $\phi \chi$ bound states, with an interaction caused by the exchange of a massless particle. But, as we know from the discussion of the Wick-Cutkosky model in the last section, the Regge trajectory does not "see" the mass of the exchanged particle, so that the interaction might as well originate from the exchange of a $\phi$-particle with mass $m$, as one would have expected from the diagram (18).

The quantity $\left(\mu / m_{r d}\right)$ that played a crucial rôle for the estimates (10)-(13), has to be replaced by $1+(m / \mu)$ in the present situation. Consequently, no bound states are expected to exist for couplings $e^{2} \lesssim 1+(m / \mu)$, and on account of the missing dependence of the Regge trajectory on the mass of the exchanged particle, our results cannot be expected to be accurate unless $e^{2} \gg 1+(m / \mu)$ (for $l=0$ ), in which case higher loop corrections will probably be important for quantitatively correct results. Thus it might appear that the results presented here for the charge one sector are of little use. However, the nature of the present study is exploratory, and it is legitimate to inquire whether other qualitative and

\footnotetext{
${ }^{3}$ We thank Norbert E. Ligterink for discussions on the asymmetric mass limit in the Bethe-Salpeter equation.
} 
quantitative features are correctly reproduced in the Regge approach. It will turn out that this is indeed the case and suggests that one may consider the spectrum following from (17) as a valid first approximation to the real spectrum, at least for $e^{2} \gtrsim 1+(\mathrm{m} / \mu)$.

We will compare our results for the bound state masses in the different charge sectors in section 5. Here, in order to further analyse the physical situation and the properties of the charge one bound states, we will formally consider the non-relativistic limit. In particular, the relation between the Regge signatures and the attractive or repulsive nature of the associated potentials will become obvious. We will start from the Bethe-Salpeter equation, which is easily written down in the ladder approximation for the present situation. In Euclidean momentum space, we have

$$
\left(p_{1}^{2}+m^{2}\right)\left(p_{2}^{2}+\mu^{2}\right) \Phi\left(p_{1}, p_{2}\right)=\int \frac{d^{4} q_{1}}{(2 \pi)^{4}} \frac{d^{4} q_{2}}{(2 \pi)^{4}} V\left(p_{1}, p_{2} ; q_{1}, q_{2}\right) \Phi\left(q_{1}, q_{2}\right)
$$

where the Bethe-Salpeter kernel is given in this approximation by

$$
\left.V\left(p_{1}, p_{2} ; q_{1}, q_{2}\right)=\right\}_{q_{1}}^{p_{2}} \rightarrow\left\{_{q_{2}}^{p_{1}}=\frac{g^{2}}{\left(p_{2}-q_{1}\right)^{2}+m^{2}}(2 \pi)^{4} \delta\left(p_{1}+p_{2}-q_{1}-q_{2}\right) .\right.
$$

Reintroducing the speed of light $c$ and taking the limit $c \rightarrow \infty$ [2] leads (after a Wick rotation to Minkowski space) to the Schrödinger equation

$$
-\frac{1}{2 m_{r d}} \nabla^{2} \psi(\mathbf{r})-e^{2} \frac{e^{-m r}}{r} \psi(-\mathbf{r})=E \psi(\mathbf{r})
$$

in the center-of-mass frame. After separation of the angular part, one is left with the Schrödinger equation for the radial part,

$$
\left(-\frac{1}{2 m_{r d}} \frac{d^{2}}{d r^{2}}+\frac{l(l+1)}{2 m_{r d} r^{2}}-(-1)^{l} e^{2} \frac{e^{-m r}}{r}\right) R(r)=E R(r),
$$

for angular momentum $l$. The presence of the factor $(-1)^{l}$ can be traced directly to the exchange form of the kernel (20). It is then clear that we get a repulsive potential for $l$ odd, corresponding to the odd signature trajectory, and an attractive one for $l$ even, corresponding to even signature, in accordance with the prediction of Regge theory. No solutions of eq. (19) are expected to exist in the non-relativistic regime. If, however, one puts $m$ equal to zero by hand in the potential term (which means neglecting the mass of the exchanged particle), the spectrum following from (22) is identical to the one obtained from $\alpha_{1}^{+}(s)$ in the limit $e^{2} \ll 1$.

To summarize, the Regge approach captures the qualitative and quantitative features of the physical situation, with the exception of those related to the mass of the exchanged particle.

\section{Charge Zero Bound States: Equal Masses}

The exchange of charge zero Reggeons turned out to lead to a very interesting set of leading Regge trajectories in ref. [5]. We found two trajectories of positive signature with a 
complicated analytical structure,

$$
\alpha_{0, \pm}^{+}(s)=\frac{g^{2} K_{m m}(s)}{2}\left(1 \pm\left(1+8 \frac{K_{\mu \mu}(s)}{K_{m m}(s)}\right)^{\frac{1}{2}}\right)-1
$$

and one trajectory of negative signature,

$$
\alpha_{0}^{-}(s)=g^{2} K_{m m}(s)-1 \text {. }
$$

The latter trajectory is identical in its functional form to $\alpha_{2}^{+}$, whereas the signatures are different. This can be easily understood by considering the contributing ladder diagrams

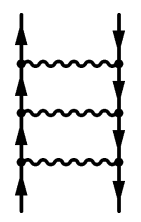

in the non-relativistic limit. There can be no contribution with two $\chi$ particles on the sides of the ladder because their Bose statistics is incompatible with the negative signature (odd angular momentum) of the trajectory. The remaining diagrams (25) then lead to the same asymptotic behaviour as the ones for charge-two Reggeons, whilst the latter can only have positive signature (even angular momentum) for the same reason.

The positive-signature sector has a more interesting structure, and we were in fact at first surprised to obtain two different trajectories in this case, one corresponding to an attractive and the other to a repulsive potential. Again, however, this qualitative feature can be better understood by considering the non-relativistic limit of the corresponding Bethe-Salpeter equation.

The typical ladder diagrams contributing to the Regge trajectory (23) are of the form

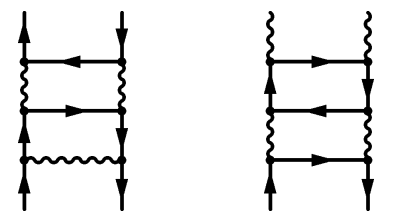

The Bethe-Salpeter equation that reproduces these diagrams most naturally has a matrix structure to take care of the possible conversion of $\phi^{\dagger} \phi$-states to $\chi \chi$-states. Such a matrix, or "multi-channel", Bethe-Salpeter equation has been used before in ref. [16 for a (fictitious) coupled channel analysis and more recently in ref. [17]. It is also implicit in our earlier results [5] where it was equivalent to implementing a matrix renormalization.

We will write the Bethe-Salpeter wave function as

$$
\Phi\left(p_{1}, p_{2}\right)=\left(\begin{array}{c}
\Phi_{1}\left(p_{1}, p_{2}\right) \\
\Phi_{2}\left(p_{1}, p_{2}\right)
\end{array}\right)
$$

where the upper entry refers to the $\phi^{\dagger} \phi$-component and the lower one to the $\chi \chi$-component of the wave function. In particular, $\Phi_{2}$ has to be symmetric under interchange of its arguments because of the Bose statistics for the $\chi$ particles. 
In order that the ladder approximation to the Bethe-Salpeter equation reproduce the diagrams (26), we take for the two-particle propagator (in Euclidean momentum space)

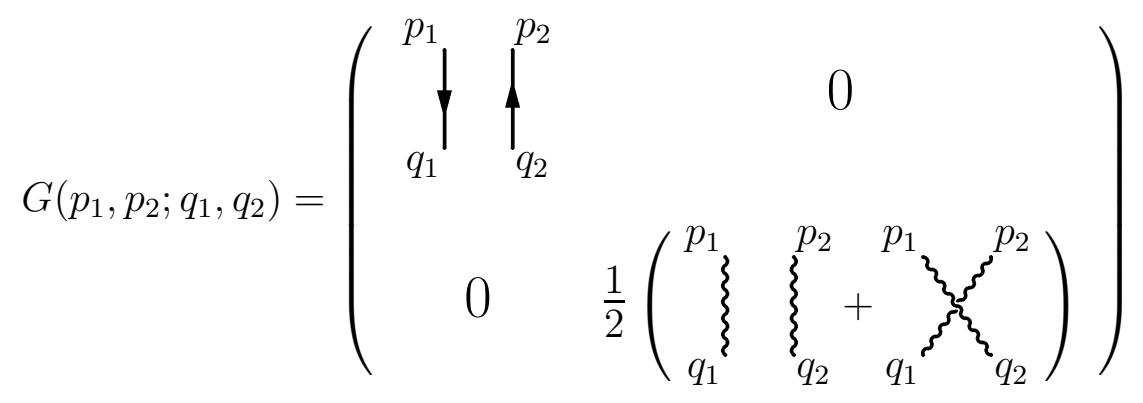

and for the Bethe-Salpeter kernel to lowest order

$$
V\left(p_{1}, p_{2} ; q_{1}, q_{2}\right)=\left(\begin{array}{ccc}
p_{1} & p_{q_{1}}^{p_{2}}
\end{array}\right)
$$

The additional factor of $1 / 2$ in the kernel is due to phase space reduction as a result of the identity of the $\chi$ particles and could equally have been put in front of the other off-diagonal element of $V$, or as an additional factor in front of the $\chi \chi$-propagator in $G$. In the present form the non-relativistic limit turns out to have a particularly transparent interpretation.

The Bethe-Salpeter equation in the ladder approximation now takes the following form,

$$
\begin{aligned}
\left(p_{1}^{2}+m^{2}\right) & \left(p_{2}^{2}+m^{2}\right) \Phi_{1}\left(p_{1}, p_{2}\right) \\
= & g^{2} \int \frac{d^{4} q_{1}}{(2 \pi)^{4}} \frac{1}{\left(p_{1}-q_{1}\right)^{2}+\mu^{2}} \Phi_{1}\left(q_{1}, p_{1}+p_{2}-q_{1}\right) \\
& \quad+\frac{g^{2}}{2} \int \frac{d^{4} q_{1}}{(2 \pi)^{4}}\left(\frac{1}{\left(p_{1}-q_{1}\right)^{2}+m^{2}}+\frac{1}{\left(p_{2}-q_{1}\right)^{2}+m^{2}}\right) \Phi_{2}\left(q_{1}, p_{1}+p_{2}-q_{1}\right) \\
\frac{1}{2}\left(p_{1}^{2}+\mu^{2}\right) & \left(p_{2}^{2}+\mu^{2}\right)\left[\Phi_{2}\left(p_{1}, p_{2}\right)+\Phi_{2}\left(p_{2}, p_{1}\right)\right] \\
= & g^{2} \int \frac{d^{4} q_{1}}{(2 \pi)^{4}}\left(\frac{1}{\left(p_{1}-q_{1}\right)^{2}+m^{2}}+\frac{1}{\left(p_{2}-q_{1}\right)^{2}+m^{2}}\right) \Phi_{1}\left(q_{1}, p_{1}+p_{2}-q_{1}\right)
\end{aligned}
$$

As in the previous section, for the purposes of a further understanding of the physical situation we will consider these equations in the non-relativistic limit, in their Wick-rotated form in Minkowski space. Due to the possible conversions between $\phi^{\dagger} \phi$-states and $\chi \chi$-states one requires that $\mu=m$ in order to have a consistent non-relativistic limit for both kinds of states.f However, we will continue to use different notations for the two masses in the following.

\footnotetext{
${ }^{4}$ Actually, the condition $|\mu-m| \ll m$ would be sufficient for this purpose. We will comment on this case below.
} 
In the formal limit $c \rightarrow \infty$, eqs. (30) lead to the following coupled Schrödinger equations, written in the center-of-mass frame,

$$
\begin{aligned}
-\frac{1}{m} \nabla^{2} \psi_{1}(\mathbf{r})-\frac{g^{2}}{16 \pi m^{2}}\left(\frac{e^{-\mu r}}{r} \psi_{1}(\mathbf{r})+\frac{1}{2} \frac{e^{-m r}}{r}\left[\psi_{2}(\mathbf{r})+\psi_{2}(-\mathbf{r})\right]\right) & =E \psi_{1}(\mathbf{r}) \\
-\frac{1}{2 \mu} \nabla^{2}\left[\psi_{2}(\mathbf{r})+\psi_{2}(-\mathbf{r})\right]-\frac{g^{2}}{16 \pi \mu^{2}} \frac{e^{-m r}}{r}\left[\psi_{1}(\mathbf{r})+\psi_{1}(-\mathbf{r})\right] & =\frac{E}{2}\left[\psi_{2}(\mathbf{r})+\psi_{2}(-\mathbf{r})\right]
\end{aligned}
$$

(the reduced masses are given by $m / 2$ and $\mu / 2$ in this case). Note that only the even component of the wave function $\psi_{2}$ appears, corresponding to the part of $\Phi_{2}$ which is symmetric under exchange of the $\chi$ particles. Consequently, the system (31) has very different characteristics for even and odd angular momentum.

To begin with the simpler case: for odd angular momentum the second equation in (31) becomes trivial and the contribution of $\psi_{2}$ to the first equation cancels, so that one is left with the Schrödinger equation for a Yukawa potential,

$$
-\frac{1}{2 m_{r d}} \nabla^{2} \psi_{1}(\mathbf{r})-e^{2} \frac{e^{-\mu r}}{r} \psi_{1}(\mathbf{r})=E \psi_{1}(\mathbf{r})
$$

the definitions of $e^{2}$ and $m_{r d}$ being as in eqs. (5) and (9), with $m_{1}=m_{2}=m$. Hence the sitation is exactly as in section 2 for the Wick-Cutkosky model, which is what we had concluded before from a consideration of the diagrams alone. Let us remark that, in analogy with the case of para-positronium, a decay into (or mixing with) states of three $\chi$-particles is possible, but needs the consideration of higher loop orders in the Regge trajectory.

For even angular momentum, and after separation of the angular part of the wave functions, the radial Schrödinger equation takes the form

$$
\left[-\frac{1}{2 m_{r d}} \frac{d^{2}}{d r^{2}}+\frac{l(l+1)}{2 m_{r d} r^{2}}-e^{2} \frac{e^{-\mu r}}{r}\left(\begin{array}{ll}
1 & 1 \\
2 & 0
\end{array}\right)\right]\left(\begin{array}{c}
R_{1}(r) \\
R_{2}(r)
\end{array}\right)=E\left(\begin{array}{c}
R_{1}(r) \\
R_{2}(r)
\end{array}\right),
$$

where $e^{2}$ and $m_{r d}$ are defined as before, and we have now explicitly put $\mu=m$. The matrix giving the potential is diagonalizable and has eigenvalues

$$
V_{+}(r)=-2 e^{2} \frac{e^{-\mu r}}{r}, \quad V_{-}(r)=+e^{2} \frac{e^{-\mu r}}{r} .
$$

Consequently, for one mixture of $\phi^{\dagger} \phi$ - and $\chi \chi$-states one has an attractive potential with bigger effective coupling constant while for the other mixture the effective potential is repulsive. The estimates of eqs. (10)-(13) applied to the present case imply that there will be no bound states for $e^{2} \lesssim 1$, in particular eq. (30) is not expected to have solutions in the non-relativistic regime.

We now compare these results with the Regge trajectories (23), which for $\mu=m$ become

$$
\alpha_{0,+}^{+}(s)=2 g^{2} K_{m m}(s)-1, \quad \alpha_{0,-}^{+}(s)=-g^{2} K_{m m}(s)-1 .
$$

The first of these leads to a Coulomb spectrum with $e^{2}$ replaced by $2 e^{2}$ in (16), while the second trajectory is identical with $\alpha_{1}^{-}$in (17) for $\mu=m$ and corresponds to a repulsive 
potential. Hence, if we simply neglect the mass in the exponential, the spectrum of bound states coincides with the spectrum generated by $V_{+}$in (34). Furthermore, the Schrödinger equation (22), which has been shown to correspond to $\alpha_{1}^{-}$, is the same as the one for the potential $V_{-}$.

Of course, we are not entitled to neglect the mass of the exchanged particle unless $e^{2} \gg 1$, and then higher order corrections give potentially important contributions. However, one again arrives at the conclusion that the Regge trajectories describe the physical situation correctly, except for the effect of the mass of the exchanged particle.

An interesting side issue is related to the fact that the Hamiltonian in (33) is apparently non-hermitian. The natural scalar product of two non-relativistic two-component wave functions $\psi\left(\mathbf{r}_{1}, \mathbf{r}_{2}\right)$ and $\psi^{\prime}\left(\mathbf{r}_{1}, \mathbf{r}_{2}\right)$ depending on the positions of the respective two particles, is given by

$$
\begin{aligned}
& \int d^{3} r_{1} d^{3} r_{2}\left(\psi_{1}^{*}\left(\mathbf{r}_{1}, \mathbf{r}_{2}\right) \psi_{1}^{\prime}\left(\mathbf{r}_{1}, \mathbf{r}_{2}\right)+\frac{1}{2} \psi_{2}^{*}\left(\mathbf{r}_{1}, \mathbf{r}_{2}\right) \psi_{2}^{\prime}\left(\mathbf{r}_{1}, \mathbf{r}_{2}\right)\right) \\
= & \int d^{3} r_{1} d^{3} r_{2} \psi^{\dagger}\left(\mathbf{r}_{1}, \mathbf{r}_{2}\right)\left(\begin{array}{cc}
1 & 0 \\
0 & 1 / 2
\end{array}\right) \psi^{\prime}\left(\mathbf{r}_{1}, \mathbf{r}_{2}\right),
\end{aligned}
$$

due to the identity of the $\chi$ particles. For this scalar product, the condition of hermiticity for an operator $H$ reads

$$
\left(\begin{array}{cc}
1 & 0 \\
0 & 1 / 2
\end{array}\right) H=H^{\dagger}\left(\begin{array}{cc}
1 & 0 \\
0 & 1 / 2
\end{array}\right),
$$

and one can easily check that the Hamiltonian in (33) is indeed hermitian in this sense.

\section{Charge Zero Bound States: Unequal Masses}

In this section, we will comment on the case of different masses $\mu \neq m$ in the charge zero sector. As shown in ref. [5], in this case there are two thresholds at $s=4 \mu^{2}$ and $4 \mathrm{~m}^{2}$, and the trajectory $\alpha_{0,+}^{+}$leads to a series of bound states (below both thresholds) and a series of resonances (between the thresholds).

An analysis of the equation

$$
\alpha_{0,+}^{+}\left(M^{2}\right)=l
$$

in the weak-coupling limit

$$
e^{2}=\frac{g^{2}}{16 \pi m^{2}} \rightarrow 0
$$

in analogy to the analysis of eq. (4) in section 2 , leads to possible solutions in the two cases $(E<0)$

$$
M=2 m+E, \quad|E| \ll m
$$

\footnotetext{
${ }^{5}$ We thank Jaime Besprosvany for drawing our attention to this point.
} 
and

$$
M=2 \mu+E, \quad|E| \ll \mu .
$$

In the first case, i.e. near the threshold $4 m^{2}$, the spectrum reduces to the Coulomb spectrum (16), with $m_{r d}=m / 2$, as long as $e^{4} \ll|m-\mu| / m$. There is a correction term of higher order in $e^{2}$, which is real for $m<\mu$ and complex for $m>\mu$. This complex value of $E$ indicates the instability of the corresponding resonance state. The sign of the imaginary part is in agreement with expectations, whereas for its size one would have expected a higher power of the effective coupling constant from semi-phenomenological considerations. Despite this descrepency, it is a highly attractive feature of the Regge methodology that it describes bound states and resonances on the same footing, thereby (in principle) furnishing information on the decay of the latter, and may play an important rôle in future applications.

Somewhat exotic, or maybe pathological, features arise when one considers the limit $m^{2} \gg \mu^{2}$ (still in the case $\left.(40)\right)$. The spectrum, which remains Coulombic as long as $e^{2} \ln \left(m^{2} / \mu^{2}\right) \ll 1$, takes the form

$$
E=\frac{m_{r d} e^{4}}{2(l+1)^{2}}\left(1-\frac{e^{2}}{\pi(l+1)} \ln \frac{m^{2}}{\mu^{2}}\right)^{2}
$$

provided that

$$
\frac{2 e^{2}}{\pi} \ln \frac{m^{2}}{\mu^{2}} \leq l+1 .
$$

For larger values of $e^{2} \ln \left(m^{2} / \mu^{2}\right)$, the solutions have a large imaginary part. Hence in this somewhat artificial limit the situation may arise that a series of resonances exist with angular momenta above a certain minimal value, while states corresponding to lower angular momenta are too unstable to be interpretable as propagating (and decaying) particles. At the present stage of the investigation, it is impossible to tell if there is any situation in the real world where this kind of behaviour should be expected.

In the case (41), near the other threshold $4 \mu^{2}$, the spectrum for the bound state region $\mu<m$ has the form

$$
E=-\frac{m_{r d} e^{8}}{2(l+1)^{4}}\left(\frac{2}{\pi} \frac{m^{4}}{\mu^{4}} \sqrt{\frac{\mu^{2}}{m^{2}-\mu^{2}}} \arctan \sqrt{\frac{\mu^{2}}{m^{2}-\mu^{2}}}\right)^{2},
$$

where now $m_{r d}=\mu / 2$, provided that $e^{4} \ll|m-\mu| / m$. Interestingly enough, this is qualitatively different from the Coulomb spectrum, and the complicated expression in parentheses

\footnotetext{
${ }^{6}$ By "semi-phenomenological considerations" we refer to the calculation of the imaginary part as it has been performed for example for positronium, using Fermi's Golden Rule. In this case one argues that the decay amplitude is proportional to the square of the overlap between the (non-relativistic) wave functions of the positron and electron. This overlap factor leads to a high power of the coupling constant in the decay rate.
} 

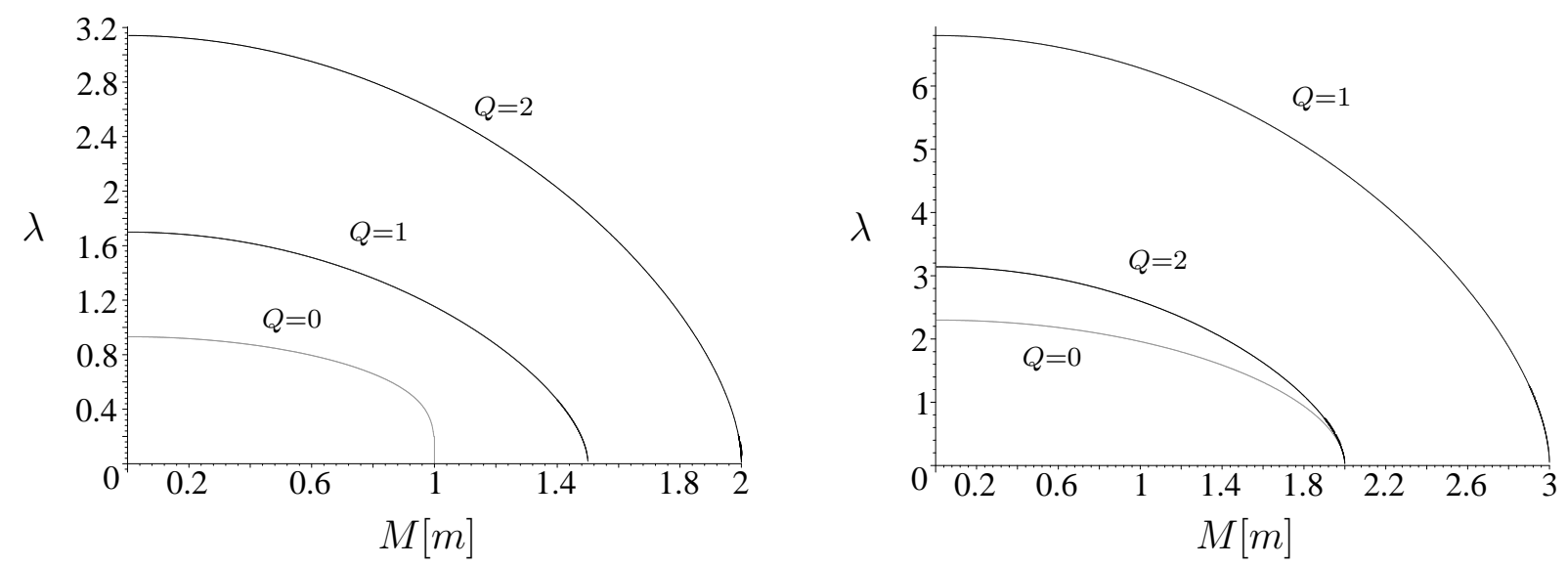

Figure 3: Graph of the coupling $\lambda=g^{2} /(16 \pi)$ versus masses of the different ground states in the sectors with charges $Q=0,1$ and 2 for $m=2 \mu$ (left) and $m=\mu / 2$ (right), respectively.

in (44) provides a $\left(\mathrm{m}^{2} / \mu^{2}\right)$-dependent modification of the effective coupling constant. In the case $m^{2} \gg \mu^{2}$, the spectrum becomes

$$
E=-\frac{2 m_{r d}}{\pi^{2}(l+1)^{4}}\left(\frac{m e^{2}}{\mu}\right)^{4},
$$

indicating that one should rather consider

$$
\frac{m e^{2}}{\mu}=\frac{g^{2}}{16 \pi m \mu}
$$

as the effective coupling constant in this case. Finally, there are no physical states in the "resonance region" $\mu>m$, since the imaginary part of the Regge trajectory is too large to interpret the corresponding poles of the scattering amplitude as propagating particles.

It will be interesting to further investigate the case of only slightly different masses $|m-\mu| \ll m$, where the condition $e^{4} \ll|m-\mu| / m$ may or may not be fulfilled. In the latter instance, the spectrum can be obtained by solving a quartic equation, hence an analytic, although certainly involved, expression can still be given. The case $|m-\mu| \ll m$ allows for a non-relativistic limit for the $\phi$ and the $\chi$ particles (see the footnote on page 10), and so should make a comparison with the corresponding Bethe-Salpeter equation in its nonrelativistic limit possible. This, in turn, could shed some light on the understanding of the results found above, as well as provide a description of the "crossover" to the case of equal masses $\mu=m$. We hope to come back to this issue in the near future.

We have also solved eqs. (4) for $m_{1}=m_{2}=m$, (38) and the corresponding equation for the charge one sector numerically, for the cases $m=2 \mu$ and $m=\mu / 2$ as examples. The results for the different $(l=0)$ bound states are presented in fig. 3 . We see that the charge zero bound states for a given coupling have the lowest mass and therefore are the most tightly bound. Alternatively, for given constituent masses the charge zero bound state becomes tachyonic at a smaller coupling than the non-zero charge states. This implies that the corresponding vacuum state will be unstable most likely giving rise to a vacuum 
condensate of charge zero bound states. Note that in the case $m=\mu / 2$ the charge two bound states are less massive than their charge one counterparts. Also remark the quartic behaviour of the charge zero bound state mass near threshold for $m=2 \mu$.

\section{Conclusions}

We now have to ask how realistic these results are and what we gain by using this method? Many of the advantages of the method stem from the fact that it is intrinsically a quantum field theory methodology and that the approximations used preserve all relevant symmetries, especially crossing symmetry, and in the case of gauge fields - gauge invariance. Preserving the crossing symmetry in particular is crucial in order to obtain certain qualitative features that otherwise would be lost.

In the case of $\phi^{\dagger} \phi$-states, where $\mu<m$, an imaginary part to the spectrum was found. Hence, our methodology gives an integrated method for finding both the real and imaginary parts of the spectrum. It is well worth noting that even the fully field theoretic Bethe-Salpeter equation does not reveal this feature in the tree-level ladder approximation, unless one uses a matricial equation. Of course, if one is prepared to consider a one-loop Bethe-Salpeter kernel then imaginary parts can be derived without the benefit of a matrix formulation. However, as is well known, including loop corrections in such a kernel is far from easy.

Although the decay of unstable states could be described directly using our techniques, the magnitude of the decay rate is very different from that found by a Fermi Golden Rule treatment. In the latter approach, as mentioned, the bound state wave functions play a crucial rôle, and there appears to be an effect of the dimensional reduction in the Regge limit on these wave functions.

A further important advantage of the present method, though one that we have neither considered nor exploited here, is its ready extension to higher loop orders. As mentioned, it is notoriously difficult to solve the Bethe-Salpeter equation including loop corrections to the kernel. In the present case higher loop corrections are included in a very natural fashion as higher order corrections to the renormalization constants and their corresponding anomalous dimensions.

We compared our results with those of the Bethe-Salpeter equation for the Wick-Cutkosky model in the charge two sector. In the relativistic, strong-coupling regime we see there are significant differences between our results and those of the Bethe-Salpeter equation. It should be emphasized though that, in the absence of a "benchmark" result from an exact solution or an experiment, this in no way invalidates our approach. Calculating to two loops will give us a better idea of the size of higher order corrections. In this sense, even if these corrections are large it may well be possible to use a technique such as Padé resummation in order to obtain more non-perturbative information such as is done in calculating fixed points from beta functions.

The chief disadvantage of calculating bound state properties in terms of Regge trajectories would seem to be that, at least in the one-loop approximation, the dependence on the mass of the "rung" particles is absent. It is for this reason that the corresponding spectra look Coulomb-like rather than Yukawa-like. Of course, this would not be a problem for a theory where the relevant exchange particles are massless. Neither does it seem to be a problem, 
as indicated in fig. 1, for sufficiently strong coupling. It remains to be seen whether higher order calculations ameliorate this problem.

In the charge one and charge zero sectors we found no corresponding Bethe-Salpeter results with which to compare. However, to gain a better insight into the physical situation and the significance of our results, we found it convenient to consider the non-relativistic limits of the corresponding Bethe-Salpeter equations, leading to different types of Schrödinger equations. The upshot of these investigations is that the Regge trajectories reproduce the qualitative and quantitative features of the situation, except for their independence of the masses of the exchanged particles.

In the absence of other results, it was interesting to compare the bound state masses in the different charge sectors with one another. We saw that, in the cases considered, charge zero bound states for a given coupling have the lowest mass and therefore are the most tightly bound. A consequence of this is that for given constituent masses the uncharged sector becomes tachyonic at a smaller coupling than the charged sector. This implies that the corresponding vacuum state will be unstable most likely giving rise to a vacuum condensate of charge zero bound states.

We may also compare with the Wilsonian RG approach of ref. [3]. There, for the WickCutkowsky model, it was not possible to access the non-relativistic regime, something which

a priori is clearly not a problem in our approach. Additionally, it would be more difficult to include higher order effects, such as the six-point function, in the Wilsonian approach than in the present one where two loop effects, though tedious, should be relatively straightforward.

In summary, we have presented a new method for computing bound state properties based on RG calculations of Regge trajectories. We found a rich bound state structure in the simple $\phi^{\dagger} \phi \chi$ theory considered here. Having shown the feasibility of such calculations, the task now is to consider more realistic theories and evaluate the importance of higher order terms. For the latter, it would be of great interest to compare the results of our approach and those of the Bethe-Salpeter equation in the context of an exact model where one may see the deviations from a known result present in the two different methodologies.

\section{References}

[1] E.E. Salpeter and H.A. Bethe, Phys. Rev. 84 (1951) 1232;

M. Gell-Mann and F. Low, Phys. Rev. 84 (1951) 350.

[2] N. Nakanishi, Prog. Phys. (Suppl.) 43 (1969) 1.

[3] U. Ellwanger, Z. Phys. C58 (1993) 619; ibid. C62 (1994) 503;

U. Ellwanger and C. Wetterich, Nucl. Phys. B423 (1994) 137.

[4] W.E. Caswell and G.P. Lepage, Phys. Lett. B167 (1986) 437.

[5] C.R. Stephens, A. Weber, J.C. López Vieyra and P.O. Hess, Phys. Lett. B414 (1997) 333; Int. J. Mod. Phys. A15 (2000) 1773.

[6] Yu.V. Novozhilov, "Introduction to Elementary Particle Theory", Pergamon Press, Oxford 1975; 
P.D.B. Collins, "An Introduction to Regge Theory and High Energy Physics", Cambridge University Press 1977.

[7] M. Derrick et al., Zeus Collaboration, Z. Phys. C69 (1996) 607;

C. Adloff et al., H1 Collaboration, Nucl. Phys. B497 (1997) 3;

J. Breitweg et al., Zeus Collaboration, Eur. Phys. J. C6 (1999) 239;

C. Adloff et al., H1 Collaboration, Nucl. Phys. B538 (1999) 3.

[8] J.C. Polkinghorne, J. Math. Phys. 4 (1963) 503;

P.G. Federbush and M.T. Grisaru, Ann. Phys. 22 (1963) 263.

[9] B.W. Lee and R.F. Sawyer, Phys. Rev. 127 (1962) 2266.

[10] L.N. Lipatov, Sov. J. Nucl. Phys. 23 (1976) 338;

V.S. Fadin, E.A. Kuraev and L.N. Lipatov, Phys. Lett. B60 (1975) 50;

E.A. Kuraev, L.N. Lipatov and V.S. Fadin, Sov. Phys. JETP 44 (1976) 443; ibid. 45 (1977) 199;

Ya.Ya. Balitsky and L.N. Lipatov Sov. J. Nucl. Phys. 28 (1978) 822.

[11] V.S. Fadin and L.N. Lipatov, Phys. Lett. B429 (1998) 127.

[12] D. O'Connor and C.R. Stephens, Nucl. Phys. B360 (1991) 297; Int. J. Mod. Phys. A9 (1994) 2805; Phys. Rev. Lett. 72 (1994) 506.

[13] G.C. Wick, Phys. Rev. 96 (1954) 1124;

R.E. Cutkosky, Phys. Rev. 96 (1954) 1135.

[14] E. Zur Linden and H. Mitter, Nuovo Cim. 61B (1969) 389.

[15] C. Schwartz and C. Zemach, Phys. Rev. 141 (1966) 1454.

[16] W.B. Kaufmann, Phys. Rev. 187 (1969) 2051.

[17] J. Boguszyński, H.D. Dahmen, R. Kretschmer and L. Lukaszuk, Multi-Channel BetheSalpeter Equation, hep-th/9910024 (1999). 